\title{
The Secular Evolution and Dynamical Architecture of the Neptunian Triplet Planetary System HD 69830
}

\author{
Jianghui JI ${ }^{1,2,3}$, Hiroshi Kinoshita ${ }^{4}$, Lin LIU ${ }^{5}$, Guangyu LI ${ }^{1,2}$ \\ jijh@pmo.ac.cn
}

\begin{abstract}
We perform numerical simulations to study the secular orbital evolution and dynamical structure in the HD 69830 planetary system with the best-fit orbital solutions by Lovis and coworkers (2006). In the simulations, we show that the triplet Neptunian system can be stable at least for 2 Gyr and the stability would not be greatly influenced even if we vary the planetary masses from Neptune-mass to Jupiter-mass. In addition, we employ the Laplace-Lagrange secular theory to investigate the long-term behaviors of the system, and the outcomes demonstrate that this theory can well describe and predict the secular orbital evolution for three Neptune-mass planets, where the secular periods and amplitudes in the eccentricities are in good agreement with those of the direct numerical integrations. We first reveal that the secular periods of the eccentricity $e_{1}$ and $e_{2}$ are identical about 8,300 yr, i.e., $P_{1}=P_{2} \simeq 2 \pi /\left|g_{1}-g_{2}\right|$ ( $g_{1,2}$ are respectively, the eigenfrequencies of the system), while the secular variation of $e_{3}$ for the outermost planet has a period of $\sim 18,200 \mathrm{yr}$. Moreover, we extensively explore the planetary configuration of three Neptune-mass companions with one massive terrestrial planet residing in $0.07 \mathrm{AU} \leq a \leq 1.20 \mathrm{AU}$, to examine the asteroid structure in this system. We underline that there are stable zones at least $10^{5} \mathrm{yr}$ for low-mass terrestrial planets locating between 0.3 and $0.5 \mathrm{AU}$, and 0.8 and $1.2 \mathrm{AU}$ with final eccentricities of $e<0.20$. Still, we also find that the secular resonance $\nu_{1}$ and $\nu_{2}$ arising from two inner planets can excite the eccentricities of the terrestrial bodies, and the accumulation or depletion of the asteroid belt are also shaped
\end{abstract}

\footnotetext{
${ }^{1}$ Purple Mountain Observatory, Chinese Academy of Sciences , Nanjing 210008,China

${ }^{2}$ National Astronomical Observatory, Chinese Academy of Sciences,Beijing 100012, China

${ }^{3}$ Department of Terrestrial Magnetism, Carnegie Institute of Washington, 5241 Broad Branch Road NW, Washington, DC 20015-1305

${ }^{4}$ National Astronomical Observatory, Mitaka, Tokyo 181-8588,Japan

${ }^{5}$ Department of Astronomy, Nanjing University, Nanjing 210093, China
} 
by orbital resonances of the outer planets, for example, the asteroidal gaps at 2:1 and 3:2 mean motion resonances (MMRs) with Planet C, and 5:2 and 1:2 MMRs with Planet D. In a dynamical sense, the proper candidate regions for the existence of the potential terrestrial planets or Habitable Zones (HZs) are 0.35 $\mathrm{AU}<a<0.50 \mathrm{AU}$, and 0.80 AU $<a<1.00 \mathrm{AU}$ for relatively low eccentricities, which makes sense to have the possible asteroidal structure in the system.

Subject headings: celestial mechanics-methods:n-body simulations-planetary systemsstars:individual (HD 69830, 47 Uma, 55 Cancri, HD 160691, GJ 876)

\section{Introduction}

To date, more than 200 extrasolar planets have been discovered about the nearby stars within 200 pc (Butler et al. 2006; The Extrasolar Planets Encyclopaedia) mostly by the measurements of Doppler surveys ${ }^{1}$ and transiting techniques. The increasing numbers of the extrasolar planets are greatly attributed to the increasing of precision of measurement. At present, the observational precision has been achieved to $\sim 1 \mathrm{~ms}^{-1}$ to $3 \mathrm{~ms}^{-1}$, whereas most observations currently have lower precision. The improvement of the observations will indeed induce the substantial discovery: (1)plentiful multiple systems, which $\sim 20$ multiple system involved in orbital resonance and secular interactions now have been detected; (2) much more low-mass companions around main-sequence stars (from Neptune-mass to Earth-mass, so-called super-Earths), e.g., 55 Cancri (McArthur et al. 2004), GJ 876 (Rivera et al. 2005), HD 160691 (Santos et al. 2004; Gozdziewski et al. 2006);(3)a true Solar System analog, bearing several terrestrial planets, the asteroidal structure and the dynamical environment of potentially terrestrial planets in the Habitable Zones (HZs) (Kasting et al. 1993) for the development of life; (4)the census of the systems, and the diversity of the planetary systems may provide abundant clues for theorists to more accurately model the planetary formation (Ida \& Lin 2004; Boss 2006).

Lovis et al. (2006) (hereafter Paper I) recently reported the discovery of an interesting system of three Neptune-mass planets orbiting about HD 69830 through high precision measurements with the HARPS spectrograph at La Silla, Chile. The nearby star HD 69830 is of spectral type $\mathrm{K} 0 \mathrm{~V}$ with an estimated mass of $0.86 \pm 0.03 M_{\odot}$ and a total luminosity of $0.60 \pm 0.03 L_{\odot}$ (Paper I), about $12.6 \mathrm{pc}$ away from the Sun. In addition, Beichman et al. (2005) announced the detection of a large excess owing to hot grains of crystalline silicates

\footnotetext{
${ }^{1}$ http://www.exoplanets.org and http://exoplanet.eu/
} 
orbiting the star HD 69830 and inferred that there could be probably a massive asteroid within 1 AU. Subsequently, Alibert et al. (2006) and Paper I performed lots of calculations to simulate the system and revealed that the innermost planet may possess a rocky core surrounded by a tiny gaseous envelope and probably formed inside the ice line in the beginning, whereas the two outer companions formed outside the ice line from a rocky embryo and then accreted the water and gas onto the envelope in the sequent formation process. Hence, it is important for one to understand the dynamical structure in the final assemblage of the planetary system (Asghari et al. 2004; Ji et al. 2005; Jiang \& Yeh 2006), and to investigate suitable HZs for life-bearing terrestrial planets (David et al. 2003; Fatuzzo et al. 2006; Jones et al. 2005; Haghighipour 2006) advancing the space missions (such as CoRot, Kepler and $T P F)$ aiming at detecting them, thus one of our goals is to focus on this issue (§2.3).

On the other hand, from a dynamical viewpoint, one may be concerned about the longterm stability of the system. In the present work, we further perform a large number of simulations of this system to examine the stability $(\S 2.1)$ and to understand the secular evolution by means of the secular theory (§2.2). Finally, we summary the main results and present a concise discussion in $\S 3$.

\section{Dynamical Analysis}

In this paper, we adopt the orbital parameters of the HD 69830 system provided by Paper I. In the simulations, we always take the stellar mass and the minimum planetary masses from Table 1, except where noted. To be specific, the mass of the host star is $0.86 M_{\odot}$, and the masses of three Neptunian planets are respectively, $10.2 M_{\oplus}, 11.8 M_{\oplus}$ and $18.1 M_{\oplus}$, where $\sin i=1$. We utilize N-body codes (Ji, Li \& Liu 2002) to perform numerical simulations by using RKF7(8) and symplectic integrators (Wisdom \& Holman 1991) for this system. In the numerical study, the adopted time stepsize is usually $\sim 2 \%-5 \%$ of the orbital period of the innermost planet. In addition, the numerical errors were effectively controlled over the integration timescale, and the total energy is generally conserved to $10^{-6}$ for the integrations. The typical timescale of simulations of the three-planet system is from $100 \mathrm{Myr}$ to $2000 \mathrm{Myr}$.

\subsection{The Stability of the HD 69830 Planetary System}

To examine the secular stability of this system, we numerically integrated the threeplanet system on a timescale of 2 Gyr in terms of the initial values listed in Table 1 . In 
Figure 1, a snapshot of the secular orbital evolution of three planets is illustrated, where $Q_{i}=a_{i}\left(1+e_{i}\right), q_{i}=a_{i}\left(1-e_{i}\right)$ (the subscipt $i=1,2,3$, individually, denoting Planet $\mathrm{B}$, $\mathrm{C}$ and $\mathrm{D})$ are, respectively, the apoapsis and periapsis distances. In the secular dynamics, the semi-major axis $a_{1}$ remains unchanged to be $0.0785 \mathrm{AU}$ for $2 \mathrm{Gyr}$, whilst $a_{2}$ and $a_{3}$ slightly undergo librations about 0.186 and 0.630 AU with small amplitudes over the same timescale. The variations of eccentricities during long-term evolution are followed, where $0.05<e_{1}<0.20,0.0<e_{2}<0.15$ and $0.069<e_{3}<0.078$. In Fig. 1 , we note that the time behaviors of $Q_{i}$ and $q_{i}$ again show regular motions of bounded orbits for three planets, indicating their orbits are well separated in the secular evolution due to smaller mutual interactions. In the numerical study, we find the system can be dynamically stable and last at least for 2 Gyr, and our simulations do extend and confirm the numerical exploration of 1 Gyr by Paper I.

To explore the stability of HD 69830 with respect to the variations of the planetary masses, we first fix $\sin i$ in increment of 0.1 from 0.1 to 0.9 . In the additional numerical experiments, we simply alter the masses but keep all orbital parameters (Table 1), again restart new runs of integration of three-planet system for 100 - $1000 \mathrm{Myr}$ with the rescaled masses. As a result, we find the system could remain definitely stable for the above investigated timescale with slight vibrations in semi-major axes and eccentricities for all planets. However, Paper I also pointed out that the system can even survive for the planets bearing the Jupiter-masses. To verify this, we further let $i=1.0^{\circ}, 0.8^{\circ}, 0.5^{\circ}$, then the planetary masses are in the Jupiter-mass range, and the outcomes for new integrations of $100 \mathrm{Myr}$ show that there is no chaotic behavior occurring in this system. This may also imply that the stability of the HD 69830 system is not so sensitive to the planetary masses.

Next, we come to the qualitative stability criteria for this system. Gladman (1993) analytically attained the minimum separation $\Delta$ between two planets of masses $m_{1}$ and $m_{2}$ moving on initially circular orbits required for stability,

$$
\Delta \simeq 2 \sqrt{3} R_{H}
$$

where the mutual Hill radius $R_{H}$ is defined by

$$
R_{H}=\left(\frac{m_{1}+m_{2}}{3 m_{c}}\right)^{1 / 3}\left(\frac{a_{1}+a_{2}}{2}\right) .
$$

With the orbital data in Table 1 , we then approximately estimate $\Delta_{12} \simeq 0.0135$ AU, for the couple of Planet B and C; similarly, $\Delta_{23} \simeq 0.0460 \mathrm{AU}$, for the pair of Planet $\mathrm{C}$ and D. Note this criteria are good guidelines for stability but are not definitive constraints for nearly-circular orbits. However, Chambers et al. (1996) presented a more general stability criterion that shows the initial orbital separation should be no less than about $10 R_{H}$ for 
multi-planet systems. For the Neptune-mass planets, the actual orbital separations are $\sim 10$ times of the analytical values that the stability requires, while the mutual distances between two orbits can amount to be $\sim 10 R_{H}$, even though these planets possess Jupiter masses. Therefore, from both the numerical and analytical views, we can safely conclude that the HD 69830 system is dynamically stable for the lifetime of the star.

\subsection{Secular Dynamics}

Considering a system of $N$ massive bodies of mass $m_{i}(i=1, N)$ moving about the central star of mass $m_{c}$ under their mutual gravitational forces and the attraction of the central body, if $m_{i} \ll m_{c}$, and these planets are with the orbits of small eccentricities and low inclinations, then the long-term evolution of the eccentricities and inclinations of all planets can be analytically described by classical Laplace-Lagrange (hereafter L-L) secular theory, provided that the mean motion resonance amongst them is absent. Many authors

have adopted the secular theory to study the orbital evolution of the planets in the systems, e.g., Ji et al. (2003) applied this theory to explore the dynamical evolution of three giant planets in 55 Cancri; more recently, Adams \& Laughlin (2006; and references therein) further investigated the dynamical interactions in the multiple planetary system by using secular theory and showed that the orbital eccentricities in these systems alter over secular time scales, of typical variations over $10^{3}-10^{5}$ years.

As for the HD 69830 system, there are three Neptune-mass planets orbiting the host star, assuming all planets are coplanar. Furthermore, in Table 1 we notice that the orbital eccentricities are smaller than about 0.10 , hence one may realize that the L-L secular theory can be applied to this system. As usual, the L-L secular theory tells us the story of the precession of the longitudes of periastron and that of the longitudes of node, and the variations of the amplitudes of the eccentricities and inclinations. Herein, we simply address the issue of the secular evolution of the eccentricities. Bearing this in mind, the L-L theory is an approximation to the actual motions, because it is valid only to the second order in the eccentricities and inclinations and the first order in the planetary masses, however, it may reveal substantial clues for long-term evolution of the system. Subsequently, we will use the analytical results from L-L theory to compare with the numerical outcomes from direct integrations.

The linear L-L secular solutions for the eccentricities (the eccentricity eigenvectors, see 
Murray \& Dermott 1999, MD99) can be written :

$$
\begin{aligned}
& e_{j} \sin \varpi_{j}=\sum_{i=1}^{3} e_{j i} \sin \left(g_{i} t+\beta_{i}\right) \\
& e_{j} \cos \varpi_{j}=\sum_{i=1}^{3} e_{j i} \cos \left(g_{i} t+\beta_{i}\right)
\end{aligned}
$$

where $e_{j i}, g_{i}$ and $\beta_{i}(j=1,2,3$, respectively for Companions $\mathrm{B}, \mathrm{C}$ and $\mathrm{D} ; i=1,2,3)$ are respectively, the magnitudes of eccentricity eigenvectors, the eigenfrequencies and the phases, which can be calculated from the initial orbital solutions given in Table 1 . In Table 2 are listed the magnitudes of the eccentricity eigenvectors $e_{j i}$ evaluated from the secular theory by considering all three planets. Firstly, we obtain three eigenfrequencies $g_{1}=236$ ". $02 \mathrm{yr}^{-1}$,

$g_{2}=79 " .76 \mathrm{yr}^{-1}$ and $g_{3}=8 " .49 \mathrm{yr}^{-1}$ (see Table 2) for the HD 69830 system, and these values are related to the precession of the periastron of three Neptune-mass planets with periods of 5,491 yr, 16,248 yr and 152,650 yr, which are essentially in agreement with the results of Paper I. In addition, the phases are $\beta_{1}=12.12^{\circ}, \beta_{2}=244.13^{\circ}$ and $\beta_{3}=224.56^{\circ}$, and now all constants in the right-side of Eqs. (3) and (4) are well determined, hence we can derive the analytical expressions of the eccentricities $e_{j}$ for Planet $j$ at any time $t$, and its squares are given by

$$
e_{j}^{2}=\left(\sum_{i=1}^{3} e_{j i} \sin \left(g_{i} t+\beta_{i}\right)\right)^{2}+\left(\sum_{i=1}^{3} e_{j i} \cos \left(g_{i} t+\beta_{i}\right)\right)^{2}
$$

Secondly, we estimate the period of $e_{1}$ in the secular orbital evolution using the L-L theory, from Table 2 , we note that the magnitudes of the eccentricity vectors $e_{11}, e_{12} \gg e_{13}$, and it is not difficult for one to understand that the mutual interactions of two inner planets are much greater than that of the distant outermost planet. Hence, we have

$$
e_{1}^{2} \simeq e_{11}^{2}+e_{12}^{2}+2 e_{11} e_{12} \cos \left(\left(g_{1}-g_{2}\right) t+\left(\beta_{1}-\beta_{2}\right)\right)
$$

Then, the secular period of $e_{1}$ given by analytical method is $P_{1} \simeq 2 \pi /\left(g_{1}-g_{2}\right)$, about 8,300 yr. Similarly, the secular period of $e_{2}$ can be also derived, where $P_{2} \simeq 2 \pi /\left|\left(g_{2}-g_{1}\right)\right|$, apparently, is equal to $P_{1}$. Now, we compare the analytical predictions with the numerical integrations. In Fig. 2, a snapshot is illustrated to perform the comparisons, while it can reflect the situation of total timescale of 2 Gyr. In this figure, the dash dot line denotes the variational eccentricities of numerical results, while the solid line represents those from secular theory. For the upper and middle panels, $P_{1}$ and $P_{2}$ are clearly evident and also in accordance with the corresponding long-term periods for eccentricities in the numerical results. In the meantime, the librating amplitudes for the eccentricities $e_{1}, e_{2}$ from the L-L theory (solid line), where $\left(e_{1 \min }, e_{1 \max }\right) \simeq\left(\left|e_{11}-e_{12}\right|,\left|e_{11}+e_{12}\right|\right)=(0.063,0.193)$, then $e_{1}$ is in 
the range $[0.05,0.20]$ and $e_{2}$ in $[0.0,0.15]$, are well coupled with those of the numerical results (dash dot line) by long-term integrations. Moreover, we also notice that $e_{1}$ experiences to be maximum while $e_{2}$ goes down minimum in the orbital evolution (see Fig.2), and vice versa, still this is because of the conversation of total angular momentum for the system. As aforementioned, the mutual perturbations between two inner planets are much more important than their interactions with the outer planet (see Table 2). On the other hand, in the secular evolution over $2 \mathrm{Gyr}, a_{1}$ and $a_{2}$ almost keep unchanged (Fig. 1), by ignoring Planet D, thus we have the total angular momentum $c_{1} \sqrt{1-e_{1}^{2}}+c_{2} \sqrt{1-e_{2}^{2}}=$ const $\left(c_{1,2}\right.$ are the combination constants of the planetary masses and semi-major axes), hence we obtain the maximum-minimum correlation of $e_{1}$ and $e_{2}$, de facto, such orbital evolution may again imply two coupled secular periods of the eccentricity in this system.

As to Planet D, the contributions of the innermost planet (HD $69830 \mathrm{~b}$ ) is much less than that of the intermediate companion (HD $69830 \mathrm{c}$ ), indicating the mutual interaction from Planet $\mathrm{C}$ and $\mathrm{D}$ is dominant, then we neglect the relevant eccentricity items arising from Planet $\mathrm{B}$ in (5), and the secular period of the eccentricity of the outermost planet is achieved, where $P_{3} \simeq 2 \pi /\left(g_{2}-g_{3}\right)$ about 18,200 yr. For the bottom panel in Fig. 2, one can observe the long-term eccentricity periods of $P_{3}$ of the secular theory and numerical simulations coincide with each other. Moreover, the secular theory presents the scope of $e_{3}$ in $[0.069,0.078]$, which also confirms the direct numerical simulations. Lastly, we reach the conclusion that the outcomes from L-L theory ${ }^{2}$ are in accordance with the numerical results.

\subsection{Dynamical Architecture and Potential HZs}

Modern observations by Spitzer and HST clearly exhibit that the circumstellar debris disks (e.g., AU Mic and $\beta$ Pic) are quite common in the early planetary formation (for a recent review see Werner et al. 2006), and Beichman et al. (2006) show that there are $13 \pm 3 \%$ for Kuiper Belt analogs around mature main sequence stars by Spitzer programs, further they point out that the existence of debris disks is extreme important to the resulting detection of individual planets and related to the formation and evolution of planetary systems. As mentioned previously, Beichman et al. (2005) also provide a clear evidence of the presence of the disk in HD 69830, and subsequently Paper I produce the best-fit orbital solutions of

\footnotetext{
${ }^{2}$ Adams \& Laughlin (2006) studied the secular theory (MD99) by adding leading-order correction for the general relativity (GR) and showed that GR may act to either stabilize or destabilize the system depending on the specific architecture of the planetary system. To examine whether GR may play a role in the stability of this system, we again numerically perform new runs by modifying the equations of motion including the GR corrections. The outcomes show that the system still retain stable in the presence of GR.
} 
three Neptune-mass planets with well-separated nearly-circular orbits, and meanwhile the present study confirms the secular orbital stability in this system, which may imply that the HD 69830 system is likely to be an analog of the Solar System, then to possess other terrestrial planets, and to be made of a potential asteroidal belt structure or Kuiper belt analog in the system. Hence, it deserves to make a detailed investigation from a numerical perspective.

To further investigate the dynamical structure and potential HZs in this system, we performed additional simulations with the planetary configuration of coplanar orbits of three Neptune-mass companions with one massive Earth-like planet. In this series of runs, the mass of the assumed terrestrial planet ranges from $0.01 M_{\oplus}$ to $1.0 M_{\oplus}$. And the initial orbital parameters are as follows: the numerical investigations were carried out in $[a, e]$ parameter space by direct integrations, and for a uniform grid of $0.01 \mathrm{AU}$ in semi-major axis (0.07 AU $\leq a \leq 1.20 \mathrm{AU})$ and 0.01 in eccentricity $(0.0 \leq e \leq 0.2)$, the inclinations are $0^{\circ}<I<5^{\circ}$, and the remainder angles are randomly distributed between $0^{\circ}$ and $360^{\circ}$ for each orbit, then the low-mass bodies were numerically integrated with three Neptune-mass planets in the HD 69830 system. In total, about 2400 simulations were exhaustively run for typical integration time spans from $10^{5}$ to $10^{6}$ yr (about $10^{6}-10^{7}$ times of the orbital period of the innermost planet). Our main results now follow.

In Figure 3, are shown the contours of the surviving time for Earth-like planets (Fig. 3a) and the status of their final eccentricities (Fig. 3b) for the integration over $10^{5} \mathrm{yr}$, where the horizontal and vertical axes are the initial $a$ and $e$. Fig.3a displays that there are stable zones for the low-mass planets in the regime between 0.3 and $0.5 \mathrm{AU}$, and 0.8 and $1.2 \mathrm{AU}$ with final eccentricities of $e<0.20$. Obviously, there exist unstable zones for the nearby orbits of three planets, where the planetary embryos there have short dynamical surviving time (usually less than $10^{3} \mathrm{yr}$ ) and the eccentricities can be quickly pumped up to a high value $\sim 0.9$ (see Fig. $3 \mathrm{~b}$ ), where the evolution is not so sensitive to the initial masses. In fact, these adjacent bodies are in association with many of the mean motion resonances of the Neptunian planets and the overlapping resonance mechanism (MD99) can reveal their chaotic behaviors of being ejected from the system in short dynamical lifetime, furthermore most of their orbits are within $3 R_{H}$ sphere of the Neptune-mass planets, and others are involved in the secular resonance with two inner companions.

Analogous to our Solar system, if consider the middle Planet $\mathrm{C}$ as the counterpart as Jupiter, we will have the regions of mean motion resonances: 2:1 (0.117 AU), 3:2 (0.142 AU), 3:1 (0.089 AU) and 5:2 (0.101 AU), 2:3 (0.244 AU), and in Fig. 3 we notice there indeed exist the apparent asteroidal gaps about or within above MMRs (e.g., 3:1 and 5:2 MMRs), while in the region between $0.10 \mathrm{AU}$ and $0.14 \mathrm{AU}$ for $e<0.10$, there are stable islands 
that the planetary embryos can last at least $10^{5} \mathrm{yr}$. In addition, for Planet D, most of the terrestrial planets in $0.50 \mathrm{AU}<a<0.80 \mathrm{AU}$ are chaotic and their eccentricities are excited to moderate even high values, the characterized MMRs with respect to the accumulation or depletion of the asteroid belt are 3:2 (0.481 AU), 2:1 (0.397 AU), 5:2 (0.342 AU), 4:3 (0.520 AU), 1:1 (0.630 AU), 2:3 (0.826 AU), 1:2 (1.000 AU), and our results enrich those of Paper I for massless bodies over two consecutive 1000-year intervals, showing a broader stable region beyond $0.80 \mathrm{AU}$. Note that there exist stable Trojan terrestrial bodies in a narrow stripe about 0.630 AU, involved in 1:1 MMR with Planet D, and they can survive at least $10^{6} \mathrm{yr}$ with resulting small eccentricities in the extended integrations ${ }^{3}$. The stable Trojan configurations may possibly take place in the extrasolar planetary systems, for example, Ji et al. (2005) explored such Trojan planets moving about 47 Uma, and Gozdziewski \& Konacki (2006) also argued that there may exist Trojan pairs configurations in the HD 128311 and HD 82943 systems. Recently, Ford \& Gaudi (2006) develop a novel method of detecting Trojan companions to transiting close-in extrasolar planets and argue that the terrestrial-mass Trojans may be detectable with present ground-based observatories, whereas the terrestrial Trojan planets with low eccentricity orbits about 1 AU would be probably a fascinating habitatable environment for other life-bearing worlds.

Malhotra (2004) and Ji et al. (2005) showed that the secular resonances may possibly exist in the general planetary systems, and play a part in the dynamical distributions of small bodies, then shape the asteroidal architecture as well as the orbital resonances. In this system, if consider a planetary embryo with a mass of $0.01 M_{\oplus}$ (in lunar mass), the related location for $\nu_{1}$ secular resonance originating from Planet B is $\sim 0.321 \mathrm{AU}$, where two eigenfrequencies for the terrestrial body and innermost planet are, respectively, 232 " $.33 \mathrm{yr}^{-1}$ and $235 " .94 \mathrm{yr}^{-1}$, which are obtained by the L-L secular theory, as the Earth-like planet bears the finite mass and then it may modify the values of the in situ eigenfrequencies $g_{i}$ $(i=1,2,3)$ of three Neptunian planets, thus $\nu_{i} \simeq g_{i}$, which also indicates that both planets almost share common secular apsidal precession rates in their motion. Fig. 3b exhibits that the eccentricity of the planetesimals could be excited by $\nu_{1}$ about $0.32 \mathrm{AU}$, and meanwhile the 5:2 MMR about 0.34 AU (for Planet D) drives the eccentricities to modest values,

\footnotetext{
${ }^{3}$ It would be likely that a fraction of the stable orbits become unstable over even longer timescale, however these results do reveal potential asteroid structure in this system. In a statistical sense, David et al. (2003) investigated the stability of the Earth-like planets in the binary systems and showed that the ejection simulations are chaotic in the studied parameter space; in addition, Fatuzzo et al. (2006) further focus on the characterization of the distribution of survival times for Earth-like planets orbiting a solar-type star with a stellar companion and present meaningful calculations on the width of the distributions of survival time across parameter space, and all the studies will provide helpful information of detecting such low-mass terrestrial planets in future survey.
} 
which may imply that the combination effects of the secular resonance and MMR can act as a hybrid dynamical mechanism of clearing of the small bodies in the disk. Similarly, $\nu_{2}$ resonance with respect to the intermediate companion (Planet $\mathrm{C}$ ) occurs for a lunar-size planet about $\sim 0.962 \mathrm{AU}$ at an eigenfrequency of $76 " .72 \mathrm{yr}^{-1}$, and the $\nu_{2}$ region is also close to $1: 2$ resonance with the outermost Planet $\mathrm{D}$, where the eccentricities for low-mass bodies about $1 \mathrm{AU}$ can be stimulated to moderate values in the extended time integrations of $10^{6}$ yr. Our results are in accordance with Nagasawa et al. (2005), who pointed out that the secular resonances can pass through the terrestrial region from outside to inside to excite the eccentricities of the separated planetary embryos. Note that $\nu_{3}$ arises from Planet D with an eigenfrequency of $8 " .62 \mathrm{yr}^{-1}$, which may be at work $\sim 1.488$ AU. In a word, the numerical study may suggest that there exist asteroid structure of the HD 69830 system.

\section{Summary and Discussions}

In this work, we have studied the secular dynamics and dynamical structure of HD 69830. We now summarize the main results as follows:

(1)In the simulations, we show that the triplet Neptunian system can be stable at least for 2 Gyr and that the stability would not be greatly influenced when we shift the planetary masses from Neptune-mass to Jupiter-mass. The L-L theory can well describe and predict the secular orbital evolution for three Neptune-mass planets, where both the secular periods and librating amplitudes in the eccentricities are well coincidence with those obtained from direct integrations. We first reveal that the secular periods of the eccentricity $e_{1}$ and $e_{2}$ are identical, about 8,300 yr. Account for the nature of near-circular well-spaced orbits, the HD 69830 system may be a close analog of the Solar System. Nevertheless, as well-known in our Solar System, Jupiter can play a major role as a dynamical shield to bounce and throw away most of small bodies at times and further to prevent many asteroids from colliding into the Earth (or other inner terrestrial planets) over the evolution. However, if all planets of HD 69830 are in Neptune-mass, such protection mechanism for the potential terrestrial planets would be weaker and larger masses would be helpful in building a harmonic habitable environment.

(2)Moreover, we extensively investigate the planetary configuration of three Neptunemass companions with one massive terrestrial planet in the region of $0.07 \mathrm{AU} \leq a \leq 1.20 \mathrm{AU}$ to examine the existence of the potential Earth-like planets and further to study the asteroid

structure or $\mathrm{HZ}$ in this system. We show that there are stable zones at least $10^{5} \mathrm{yr}$ for the low-mass terrestrial planets locating between 0.3 and $0.5 \mathrm{AU}$, and 0.8 and $1.2 \mathrm{AU}$ with final eccentricities of $e<0.20$. Furthermore, we also find that the secular resonance $\nu_{1}$ and $\nu_{2}$ 
arising from two inner planets can excite the eccentricities of the terrestrial bodies, and the accumulation or depletion of the asteroid belt are also shaped by orbital resonances of the outer planets, for example, the asteroidal gaps of 2:1 and 3:2 MMRs with Planet C, and 5:2 and 1:2 resonances with Planet D. On the other hand, the stellar luminosity of HD 69830 is lower than that of the Sun, thus the HZ should shift inwards compared to our Solar System. However, Kuchner (2003) pointed out that the habitable regime is considered to be centered at $a_{p} \geq 1.03 \mathrm{AU}\left(L / L_{\odot}\right)^{1 / 2}$, such that a water planet can exist in equilibrium with stellar radiation, where $a_{p}$ is the radius of the planet's orbit and $L$ is the luminosity of the host star, then $a_{p}$ is approximate to $0.80 \mathrm{AU}$ for HD 69830. Therefore, in a dynamical consideration, the proper candidate regions for the existence of the potential terrestrial planets or HZs are 0.35 $\mathrm{AU}<a<0.50 \mathrm{AU}$, and 0.80 AU $<a<1.00 \mathrm{AU}$ for relatively low eccentricities. Hopefully, the future space-based observations, e.g., CoRot, Kelper and TPF will definitely present a handful of samples belonging to the category of the terrestrial bodies. In final, we may summarize that the HD 69830 system can possess the asteroidal architecture resembling to the Solar System and both the mean motion resonance (MMR) and secular resonances (Malhotra 2004; Ji et al. 2005; Nagasawa et al. 2005) will work together to influence on the distribution of the small bodies in the planetary system. However, the formation of this system is still a mystery and needs further investigation in forthcoming study.

We would like to thank the anonymous referee for a prompt report and valuable comments and suggestions that help to improve the contents. J.H.J. is much grateful to Paul Butler and Megan Falla for their hospitality during his stay of visit at DTM and also appreciates to the astronomy group therein: Sean Solomon, Alan Boss, John Chambers, Sara Seager, Alycia Weinberger, Sandy Keiser, Vera Rubin, Mercedes Lopez-Morales, and Kevin Wang, Terry Stahl, Janice Dunlap, Alicia Case and many others for their kindness, assistance and helpful discussions. We are also thankful to Zhou Q.L. for the assistance of computer utilization. Part of the computations were carried out on high performance workstations at Laboratory of Astronomical Data Analysis and Computational Physics of Nanjing University. This work is financially supported by the National Natural Science Foundations of China (Grants 10573040, 10673006, 10203005, 10233020) and the Foundation of Minor Planets of Purple Mountain Observatory.

\section{REFERENCES}

Adams, F.C., \& Laughlin, G. 2006, ApJ, 649, 992

Alibert, Y., et al. 2006, A\&A, 455, L25 
Asghari, N., et al. 2004, A\&A, 426, 353

Beichman, C. A., et al. 2005, ApJ, 626, 1061

Beichman, C. A., et al. 2006, AAS DPS Meeting, 38, 54.01

Boss, A. P. 2006, ApJ, 644, L79

Butler, R. P., et al. 2006, ApJ, 646, 505

Chambers, J. E., et al. 1996, Icarus, 119, 261

David, E. M., et al. 2003, PASP, 115, 825

Fatuzzo, M., et al. 2006, PASP, accepted, (astro-ph/0609305)

Ford, E. B., \&, Gaudi, B. S. 2006, ApJ, submitted, (astro-ph/0609298)

Gladman, B. 1993, Icarus, 106, 247

Gozdziewski, K., \& Konacki, M. 2006, ApJ, 647, 573

Gozdziewski, K., et al. 2006, submitted to ApJ, (astro-ph/0608279)

Haghighipour, N. 2006, ApJ, 644, 543

Ida, S., \& Lin, D. N. C. 2004, ApJ, 604, 388

Ji, J. H., Li, G.Y. \& Liu, L. 2002, ApJ, 572, 1041

Ji, J. H., Kinoshita, H., Liu, L. \& Li, G.Y. 2003, ApJ, 585, L139

Ji, J. H., Liu, L., Kinoshita, H., \& Li, G.Y. 2005, ApJ, 631, 1191

Jiang, I. G., \& Yeh, L. C. 2006, ApJ, accepted, (astro-ph/0610668)

Jones, B. W., Underwood, D. R., \& Sleep, P. N. 2005, ApJ, 622, 1091

Kasting, J. F., et al. 1993, Icarus, 101, 108

Kuchner, M.J. 2003, ApJ, 596, L105

Lovis, C., et al. 2006, Nature, 441, 305 (Paper I)

Rivera, E. J., et al. 2005, ApJ, 634, 625

Malhotra, R. 2004, AAS DPS Meeting 36, 42.04 
McArthur, B.E., et al. 2004, ApJ, 614, L81

Murray, C. D., \& Dermott, S. F. 1999, Solar System Dynamics (New York: Cambridge Univ. Press) (MD99)

Nagasawa, M., Lin, D.N.C., \& Thommes, E. 2005, ApJ, 635, 578

Santos, N.C., et al. 2004, A\&A, 426, L19

Werner, M., et al. 2006, ARA\&A, 44, 269

Wisdom, J., \& Holman, M. 1991, AJ, 102, 1528 
Table 1. The orbital parameters of HD 69830 planetary system

\begin{tabular}{llll}
\hline \hline \multicolumn{1}{c}{ Parameter } & Companion B & Companion C & Companion D \\
\hline$M \sin i\left(M_{\oplus}\right)^{\mathrm{a}}$ & 10.2 & 11.8 & 18.1 \\
Orbital period $P($ days $)$ & 8.667 & 31.56 & 197 \\
$a(\mathrm{AU})$ & 0.0785 & 0.186 & 0.630 \\
Eccentricity $e$ & 0.10 & 0.13 & 0.07 \\
Longitude of Peri. $\varpi(\mathrm{deg})$ & 340 & 221 & 224 \\
Epoch of Peri.(JD) & 2453496.8 & 2453469.6 & 2453358 \\
\hline
\end{tabular}

a The parameters are taken from Paper I. The mass of the star is $0.86 M_{\odot}$.

Table 2. The values of $e_{j i}\left(\times 10^{8}\right)(j=1,2,3$, respectively for Companions B, C and D; $i=1,2,3)$ and the eigenfrequencies $g_{j}$ are given from secular Laplace-Lagrange theory for HD 69830 planetary system, and the unit for $g_{j}$ is $\mathrm{yr}^{-1}$

\begin{tabular}{rrrr}
\hline \hline$e_{j i}$ & \multicolumn{1}{c}{1} & \multicolumn{1}{c}{2} & \multicolumn{1}{c}{$3^{\mathrm{b}}$} \\
\hline$e_{1 i}$ & 12785986 & 6481193 & 387801 \\
$e_{2 i}$ & -6191399 & 7506478 & 677060 \\
$e_{3 i}$ & 67818 & -312891 & 7351714 \\
\hline$g_{j}$ & $236 " .02$ & $79^{\prime \prime} .76$ & $8^{\prime \prime} .49$ \\
\hline
\end{tabular}

${ }^{\mathrm{b}}$ Note that $e_{13}$ and $e_{23}$ terms are contributions from Planet $\mathrm{D}$, which is much less than those of the mutual effects of the two inner planets. 
Fig. 1. - Snapshot of the secular orbital evolution of three planets is shown (for $t=10^{8}$ yr), where $Q_{i}=a_{i}\left(1+e_{i}\right), q_{i}=a_{i}\left(1-e_{i}\right)$ are, respectively, the apoapsis and periapsis distances, and the straight lines are the semi-major axes $a_{i}$. Note $a_{1}$ remains unchanged to be $0.0785 \mathrm{AU}$ for $2 \mathrm{Gyr}$, and $a_{2}$ and $a_{3}$ slightly librate about 0.186 and $0.630 \mathrm{AU}$ with small amplitudes. Over the long-term evolution, the eccentricity variations are $0.05<e_{1}<0.20$, $0.0<e_{2}<0.15$ and $0.069<e_{3}<0.078$.

Fig. 2.- Comparison of secular theory with direct numerical integration for the planetary system HD 69830. The eccentricity variations produced by direct numerical integration are shown by the dash dot lines; the corresponding eccentricity variations given by secular theory are shown by the solid lines. The two approaches are in good agreement and the envelope of the variations of eccentricity are match up extremely well.

Fig. 3.- Left: Contour of the surviving time for Earth-like planets for the integration of $10^{5}$ yr. Right: Status of their final eccentricities. Horizontal and vertical axes are the initial $a$ and $e$. Stable zones for the low-mass planets in the region between 0.3 and $0.5 \mathrm{AU}$, and 0.8 and 1.2 AU with final eccentricities of $e<0.20$ (cool eccentricities). Unstable zones for the nearby orbits of three planets, and the planetary embryos there have short dynamical surviving time and the eccentricities can be quickly pumped up to a high value $\sim 0.9$. The secular resonance $\nu_{1}$ and $\nu_{2}$ can excite the eccentricities of the terrestrial bodies, and the accumulation or depletion of the asteroid belt are also shaped by orbital resonances of the outer planets, for example, the asteroidal gaps of 2:1 and 3:2 MMRs with the middle planet, and 5:2 and 1:2 resonances with the outmost planet. 


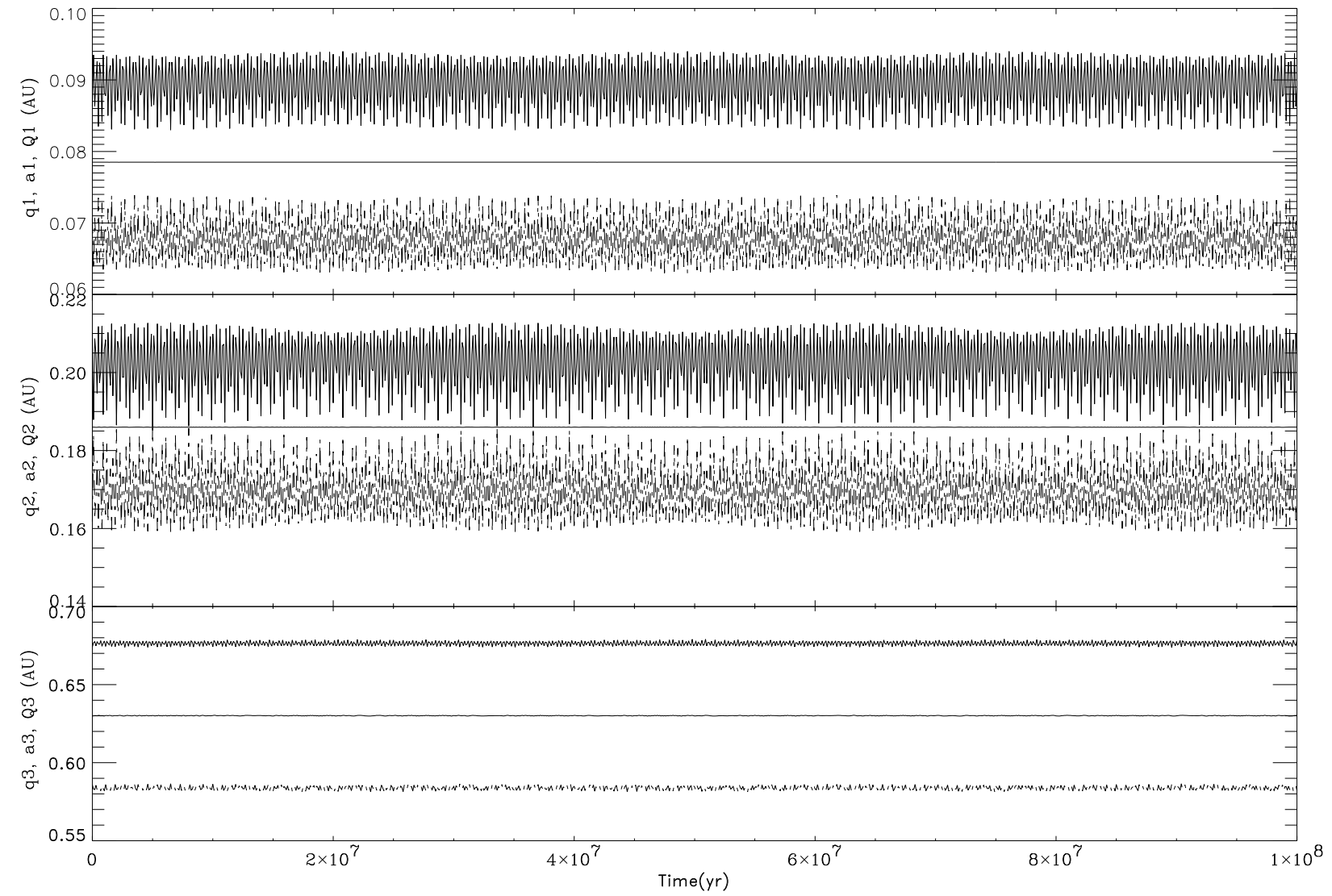

Fig. 1.- 


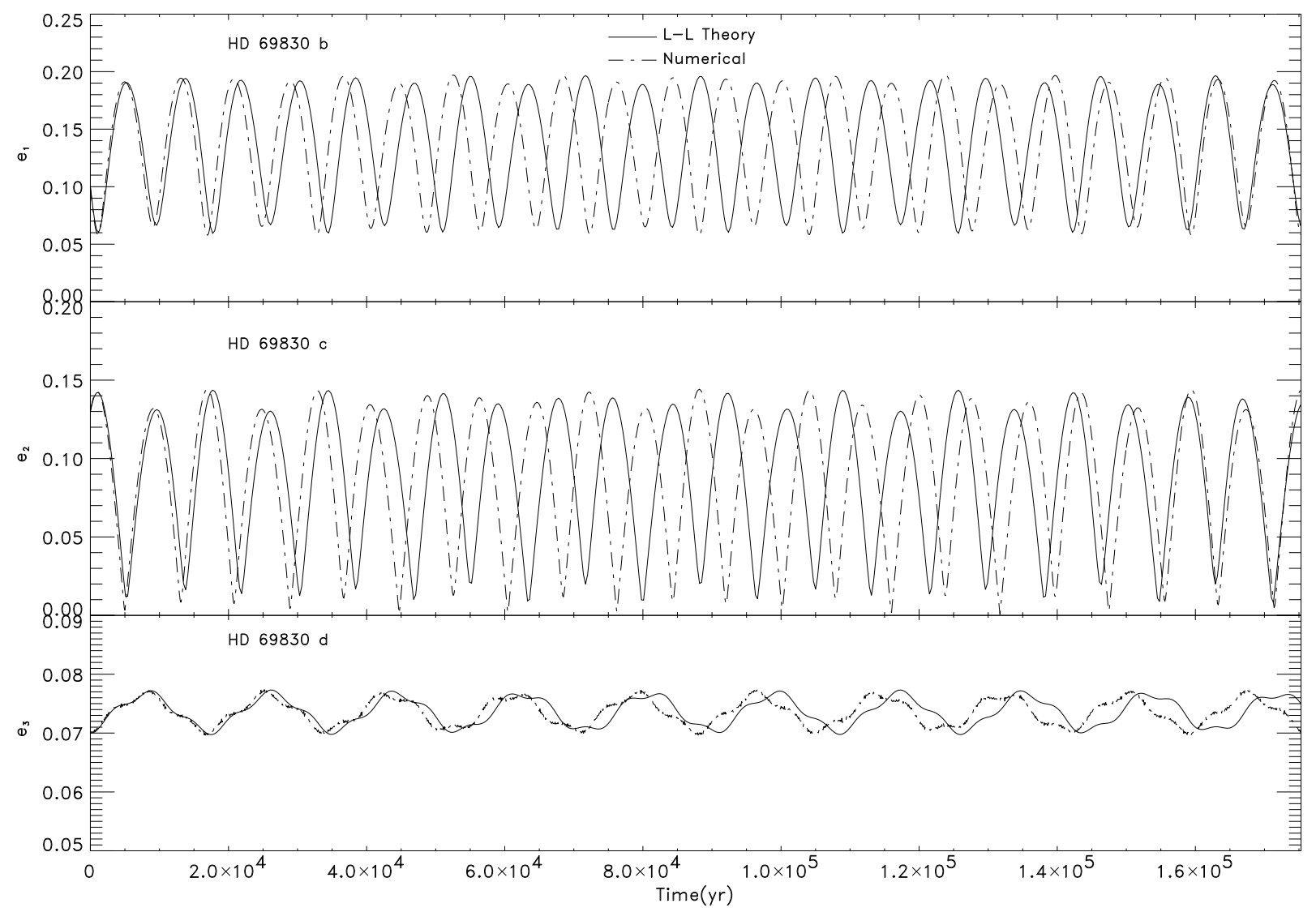

Fig. 2.- 


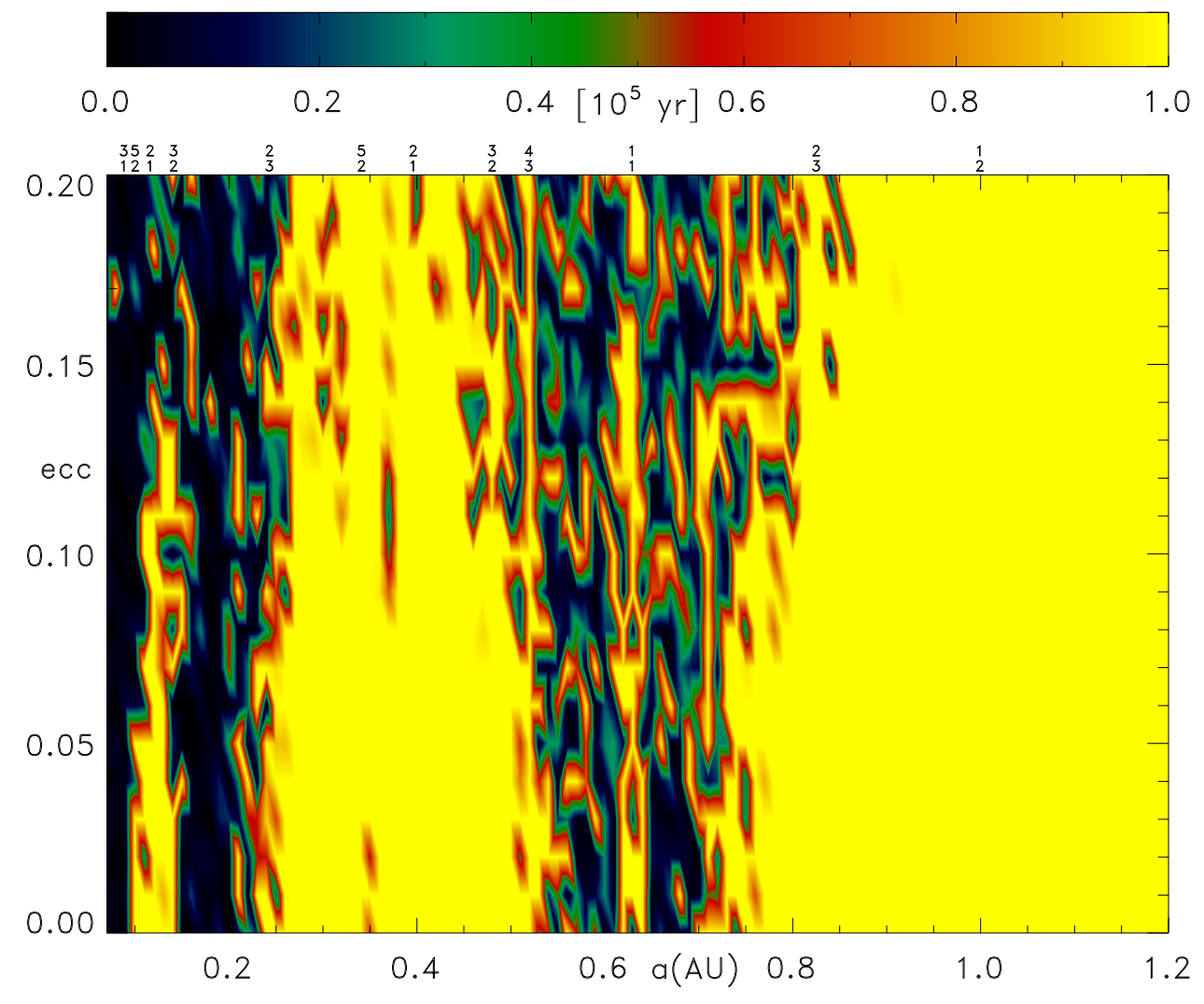

Fig. 3.- 


\section{- 19 -}

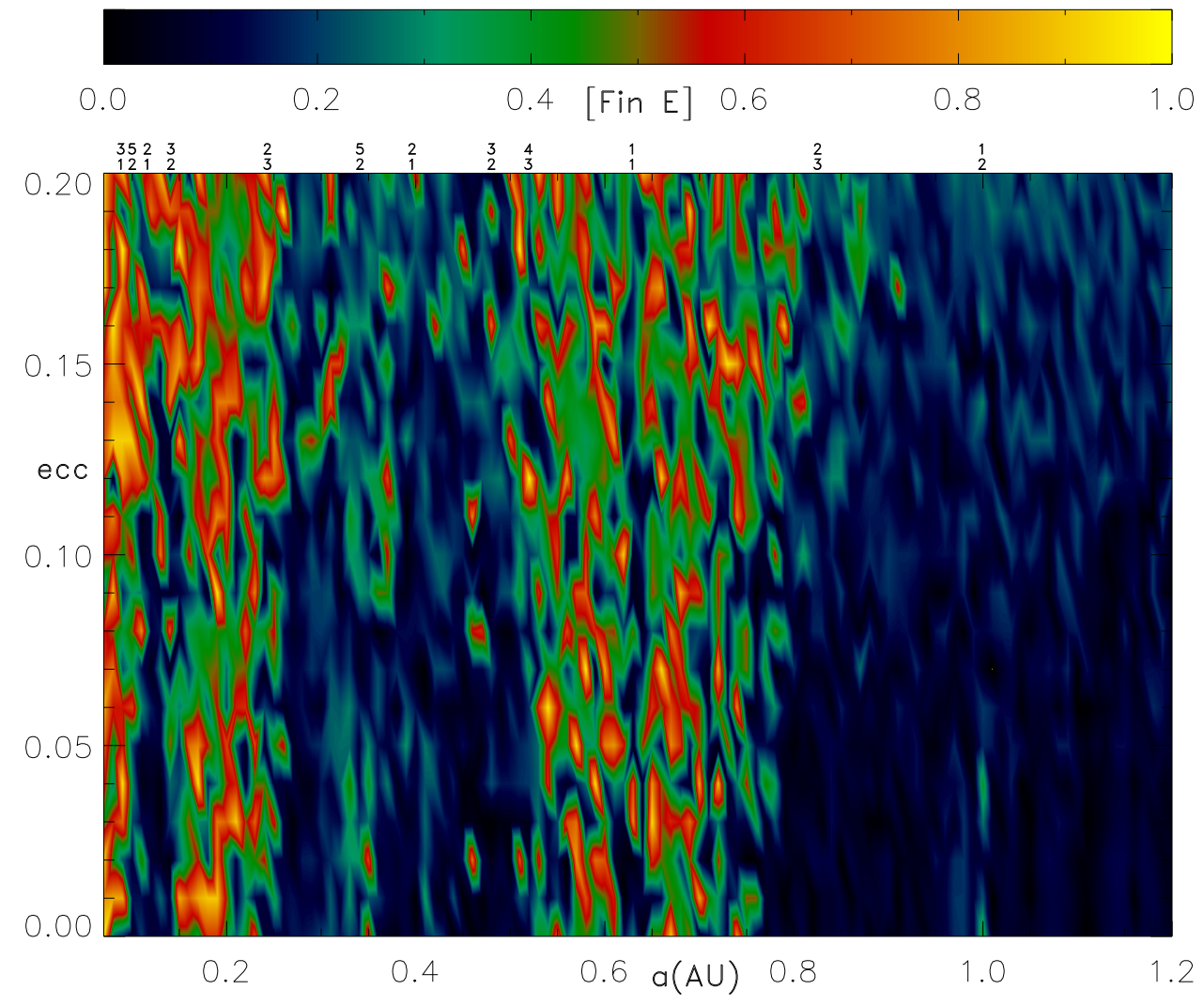

Fig. 3.- 